\title{
The Generalized Correlation for the Evaluation of the Influence of the Stefan Flow on the Heat Transfer Coefficient
}

\author{
A. P. Baskakov and O. A. Rakov \\ Ural Federal University, ul. Mira 19, Yekaterinburg, 620002 Russia
}

\begin{abstract}
The analytical equations for the steady-state heat-and-mass transfer in the steam evaporation/condensation processes from the steam-gas mixtures on the planar and spherical surfaces are derived. The vapor flow through the motionless dry gas is considered according to the method proposed by Maxwell for the solution of the diffusion problems.

The relationships for the calculation of the coefficients taking into account an increase in the mass output and an increase or a decrease in the heat emission (depending on the directions of the heat-and-mass flows) as a result of the influence of the Stefan flow are presented.

The derived relationships can be used to calculate the apparatuses in which the steam evaporation or condensation from the steam-gas mixture occurs (the coolers of the vapor from deaerators, the apparatuses for the deep utilization of the heat of the combustion products, the condensation boilers, etc.).
\end{abstract}

Keywords: Stefan flow, heat-and-mass transfer, heat conduction, diffusion, steam condensation, gas-steam mixture

DOI: $10.1134 / \mathrm{S} 0040601513080028$

The steady-state diffusion process is always associated with the formation of the diffusing substance in one place and its disappearance or the carry-over by the flow in another place.

\section{HEAT TRANSFER IN A PLANAR LAYER}

In a canonic case of the heat transfer, it is transferred through the layer of the mixture of the steam and the dry gas between two surfaces (figure). Gap between the surfaces can be considered as the thickness of the reduced film during the heat-and-mass transfer. The problem is one-dimensional, and the mixture velocity along the surface equals zero. At the left surface, the steam is injected, i.e., the left surface is the steam source. The steam diffuses to the right surface and condenses on it; thereby, the steam sink occurs here. The surfaces are impermeable for the dry gas.

It is acceptable to express diffusion flow density $j_{i}$ in the isothermal layer by the Fick law through absolute concentration gradient $\nabla c_{i}$ of the diffusing component expressed in kilograms per cubic meter $\left(\mathrm{kg} / \mathrm{m}^{3}\right)$ or kilomoles per cubic meter $\left(\mathrm{kmol} / \mathrm{m}^{3}\right)$ :

$$
j_{i}=-D \nabla c_{i},
$$

where $D$ is the diffusivity.

If $D$ is measured in square meters per second $\left(\mathrm{m}^{2} / \mathrm{s}\right)$, then the steam flow density is expressed in kilograms per square meter per second $\left[\mathrm{kg} /\left(\mathrm{m}^{2} \mathrm{~s}\right)\right]$ or kilomoles per square meter per second $\left[\mathrm{kmol} /\left(\mathrm{m}^{2} \mathrm{~s}\right)\right]$.
For the nonisothermal case, formula (1) is invalid for the calculation of the diffusion flow density since the absolute concentration of the diffusing component is temperature-independent. Most often, relative mass concentration $m, \mathrm{~kg} / \mathrm{kg}$; or relative volume concentration $r, \mathrm{~m}^{3} / \mathrm{m}^{3}$ (it coincides with the relative molar concentration for ideal gases) is used [1-5]. Let us substitute $c_{i}=m_{i} \rho$ into formula (1), where $\rho$ is the mixture density, and differentiate $j_{i}=-D \nabla\left(m_{i} \rho\right)$, considering that diffusivity is constant. We derive $j_{i}=-D \rho \nabla m_{i}-$ $D m_{i} \nabla \rho$. Equations of motion and energy are usually solved accepting $\rho=$ const for simplicity; in this case, the Fick law for the nonisothermal case is written in the form

$$
j_{i}=-\rho D \nabla m_{i}
$$

Only this record form is particularly accepted in [5].

According to the diffusion laws, the counterflows of the diffusing gases in the binary mixture are equal to one another. As applied to the scheme under consideration (figure), this means that

$$
-D \frac{d m_{\mathrm{s}}}{d y}=D \frac{d m_{\mathrm{d} . \mathrm{g}}}{d y}
$$

However, the dry gas is not produced anywhere and does not disappear; therefore, it should be motionless, which can be only under the condition that the convective (the Stefan) steam-gas mixture flow moves oppositely to the diffusion dry gas flow and compensates it. Thus, we obtain the formula for the Stefan mass flow density [5] in the form 


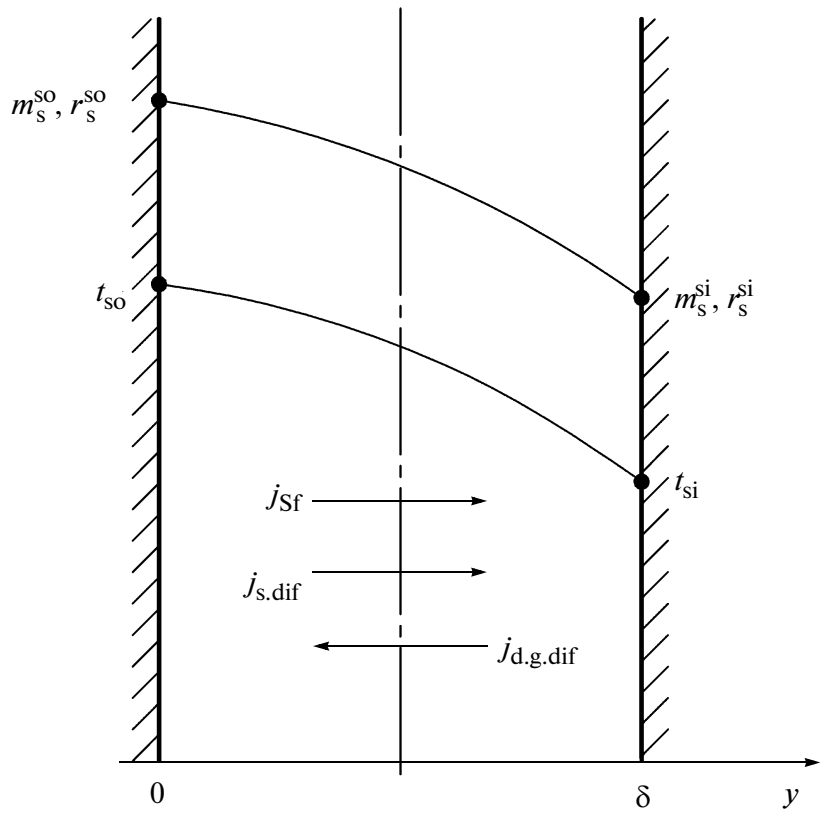

Distribution of the steam concentration and the temperature of the steam-gas mixture over the reduced film thickness with the combined occurrence of the heat-and-mass transfer.

$$
j_{\mathrm{Sf}}=-\frac{\rho D}{1-m_{\mathrm{s}}} \frac{d m_{\mathrm{s}}}{d y} .
$$

The volume (convective) velocity of this flow $w_{\mathrm{Sf}}=j_{\mathrm{Sf}} / \rho$. It should be emphasized that this velocity is determined in the reference system associated with the center of mass, or in the system, in which the opposite mass diffusion flows of the steam and the dry gas rather than volume ones, are equalized [1, 2, 4]. Since the densities of the steam-gas mixture $\rho$ and dry gas $\rho_{\text {d.g }}$ are different, the convective velocities of opposite flows $w_{\mathrm{Sf}}$ and $j_{\mathrm{d} . \mathrm{g}} / \rho_{\mathrm{d} . \mathrm{g}}$ are different. The dry gas is mobile when counting the system of the center of mass or, in other words, the center of mass moves relatively to the surfaces under consideration (figure). Consequently, the use of formulas (2) and (3), or relative mass concentrations, is not quite convenient to solve the problem depicted in the figure. One of the authors of this article had met this difficulty in [6], where rather cumbersome approximated solutions were derived.

However, since the dry gas is motionless, its diffusion and transfer by the Stefan flow can be not taken into account generally, thus considering only the steam motion relative to the motionless dry gas and the surfaces. This approach was proposed by Maxwell and widely used by Stefan and Frank-Kamenetskii [2]. The author of [4] called it the Hittorf system. In this system, the flow density of one component relative to another one, $\mathrm{kmol} /\left(\mathrm{m}^{2} \mathrm{~s}\right)$, is expressed in the form

$$
j_{\mathrm{s}}=-\frac{D}{\left(1-r_{\mathrm{s}}\right) \bar{V}_{\mu}} \frac{d r_{\mathrm{s}}}{d y},
$$

where $\bar{V}_{\mu}$ is the volume of 1 kmole mixture at its pressure and temperature and $r_{\mathrm{s}}$ is the steam volume fraction in the mixture.

Strictly speaking, the diffusivity and the molar volume depend on coordinate $y$ due to the temperature oscillations over the thickness of the reduced film, but these variations partially compensate one another. Accepting $D / \bar{V}_{\mu}=$ const, let us rewrite (4) in the form

$$
\frac{d r_{\mathrm{s}}}{1-r_{\mathrm{s}}}=-j_{\mathrm{s}} \frac{\bar{V}_{\mu}}{D} d y
$$

and, taking into account that molar steam flow $j_{\mathrm{s}}$ is independent of $y$ in the steady-state mode, we derive the differential equation with separated variables. The boundary conditions are as follows:

$$
\begin{aligned}
Y=0: & r_{\mathrm{s}}=r_{\mathrm{s}}^{\mathrm{so}} \text { and } t=t_{\mathrm{so}} ; \\
Y=1: & r_{\mathrm{s}}=r_{\mathrm{s}}^{\mathrm{si}} \text { and } t=t_{\mathrm{si}},
\end{aligned}
$$

where $Y=y / \delta$ is the dimensionless coordinate; $r_{\mathrm{s}}^{\mathrm{so}}, t_{\mathrm{so}}$ and $r_{\mathrm{s}}^{\mathrm{si}}, t_{\mathrm{si}}$ are the relative volume concentrations and temperatures of the steam source and sink. The solution of Eq. (5) has the form [2]:

$$
\frac{1-r_{\mathrm{s}}}{1-r_{\mathrm{s}}^{\mathrm{so}}}=\left(\frac{1-r_{\mathrm{s}}^{\mathrm{si}}}{1-r_{\mathrm{s}}^{\mathrm{so}}}\right)^{Y} \text {. }
$$

Derivative $d r_{\mathrm{s}} / d Y$, which is substituted into (4), is found from this formula. As a result, the formula for the steam flow density through the reduced film is derived, $\mathrm{mol} /\left(\mathrm{m}^{2} \mathrm{~s}\right)$ :

$$
j_{\mathrm{s}}=\frac{D}{\delta \bar{V}_{\mu}} \ln \frac{1-r_{\mathrm{s}}^{\mathrm{si}}}{1-r_{\mathrm{s}}^{\mathrm{so}}} .
$$

The steam flow is usually calculated by the formula $j_{\mathrm{s}}^{0}=\beta\left(c_{\mathrm{s}}^{\mathrm{so}}-c_{\mathrm{s}}^{\mathrm{si}}\right) / \mu_{\mathrm{s}}=\beta \rho_{\mathrm{s}}\left(r_{\mathrm{s}}^{\mathrm{so}}-r_{\mathrm{s}}^{\mathrm{si}}\right) / \mu_{\mathrm{s}}$, and $\beta=D / \delta$ in the case under consideration. Dividing $j_{\mathrm{s}}$ by $j_{\mathrm{s}}^{0}$ and substituting $\bar{V}_{\mu}=\mu / \rho$, we derive coefficient $K_{j}$, which takes into account an increase in the mass flow under the effect of the Stefan flow:

$$
K_{j}=\frac{\ln \frac{1-r_{\mathrm{s}}^{\mathrm{si}}}{1-r_{\mathrm{s}}^{\mathrm{so}}}}{r_{\mathrm{s}}^{\mathrm{so}}-r_{\mathrm{s}}^{\mathrm{si}}} .
$$

We further consider two cases when the directions of heat and mass flows coincide as well as when they are opposite. The steam flow does not interact with the surfaces (figure). In the first case, the steam being filtered through the motionless dry gas layer is cooled from temperature $t_{\mathrm{so}}$ to $t_{\mathrm{si}}$ partially transferring its enthalpy to the gas (we do not take into account the 
heat of the phase transition at this stage). The summary amount of the heat transferred from the steam to the gas is $j_{\mathrm{s}}\left(\mu_{\mathrm{s}} c_{\mathrm{s}}\right)_{p}\left(t_{\mathrm{so}}-t_{\mathrm{si}}\right)$. The steam transfers the additional amount of heat $q_{\mathrm{conv}}=j_{\mathrm{s}}\left(\mu_{\mathrm{s}} c_{\mathrm{s}}\right)_{p}\left(t-t_{\mathrm{si}}\right)$, through section $Y$, where $\left(\mu_{\mathrm{s}} c_{\mathrm{s}}\right)_{p}$ is the specific molar heat capacity of the steam at a constant pressure. Along with the heat flow transferred by the heat conduction, the summary heat flow is

$$
q=-\frac{\lambda}{\delta} \frac{d t}{d Y}+j_{\mathrm{s}}\left(\mu_{\mathrm{s}} c_{\mathrm{s}}\right)_{p}\left(t-t_{\mathrm{si}}\right),
$$

where $\lambda$ is the thermal conductance of the mixture.

Heat flow $q$ and substance flow $j_{\mathrm{s}}$ in the steady-state mode are independent of coordinate $Y$. Let us write Eq. (8) for $Y=0$ and $Y=1$ :

$$
-\left.\frac{\lambda}{\delta} \frac{d t}{d Y}\right|_{Y=0}+j_{\mathrm{s}}\left(\mu_{\mathrm{s}} c_{\mathrm{s}}\right)_{p}\left(t_{\mathrm{so}}-t_{\mathrm{si}}\right)=-\left.\frac{\lambda}{\delta} \frac{d t}{d Y}\right|_{Y=1} .
$$

It means that the heat conduction flow from the steam-gas mixture to the right surface (figure) is larger than from the left surface to the mixture due to the amount of heat transferred by the steam to the dry gas during its cooling from $t_{\mathrm{so}}$ to $t_{\mathrm{si}}$.

Let us introduce dimensionless coordinate $\theta=(t-$ $\left.-t_{\mathrm{si}}\right) /\left(t_{\mathrm{so}}-t_{\mathrm{si}}\right)$ and the Stefan dimensionless complex $\mathrm{Sf}=\mathrm{Sf}=j_{\mathrm{s}}\left(\mu_{\mathrm{s}} c_{\mathrm{s}}\right)_{p} \delta / \lambda$, and transform Eq. (8) to the form:

$$
d Y=\frac{d \theta}{\mathrm{Sf} \theta-\frac{q \delta}{\lambda\left(t_{\mathrm{so}}-t_{\mathrm{si}}\right)}} .
$$

Its solution for boundary condition $\theta=1$ and $Y=0$ is as follows:

$$
\ln \frac{\operatorname{Sf} \theta-\frac{q \delta}{\lambda\left(t_{\mathrm{so}}-t_{\mathrm{si}}\right)}}{\mathrm{Sf}-\frac{q \delta}{\lambda\left(t_{\mathrm{so}}-t_{\mathrm{si}}\right)}}=\operatorname{Sf} Y .
$$

Substituting $\theta=0$ at $Y=1$ into (9), after small transformations, we derive the formula for the calculation of the heat flow during the combined heat-andmass transfer:

$$
q=j_{\mathrm{s}}\left(\mu_{\mathrm{s}} c_{\mathrm{s}}\right)_{p}\left(t_{\mathrm{so}}-t_{\mathrm{si}}\right) \frac{\operatorname{expSf}}{\operatorname{expSf}-1} .
$$

After the substitution $r_{\mathrm{s}}=1-r_{\mathrm{d} . \mathrm{g}}$ and $\bar{V}_{\mu}=\mu / \rho$, in Eq. (6), where $\mu$ is the molar mass of the steam mixture with the dry gas, and the substitution of the expression for the steam flow density into the formula for determining $\mathrm{Sf}$, taking into account that $\lambda=a \rho c_{p}$, where $a$ and $c_{p}$ are the thermal diffusivity and the spe-

\footnotetext{
${ }^{1}$ This circumstance was not sufficiently taken into account in [6]; therefore, inaccuracy was committed. The difference $\left(t_{0}-t_{\infty}\right)$ instead of $t_{0}$ should stand in Eqs. (15), (16), (20), (21), and the formula for $\mathrm{Nu}$ in p. 61 [6], while simply $\left(t_{0}-t_{\infty}\right)\left(1-m_{\infty}\right)$ should be parenthesized in Eq. (17).
}

cific mass heat capacity of the mixture at a constant pressure, we write

$$
\mathrm{Sf}=\frac{D \rho\left(\mu_{\mathrm{s}} c_{\mathrm{s}}\right)_{p}}{\mu \lambda} \ln \frac{r_{\mathrm{d} . \mathrm{g}}^{\mathrm{si}}}{r_{\mathrm{d} . \mathrm{g}}^{\mathrm{so}}}=\frac{D}{a} \frac{\left(\mu_{\mathrm{s}} c_{\mathrm{s}}\right)_{p}}{\mu c_{p}} \ln \frac{r_{\mathrm{d} . \mathrm{g}}^{\mathrm{si}}}{r_{\mathrm{d} . \mathrm{g}}^{\mathrm{so}}}=A \ln \frac{r_{\mathrm{d} . \mathrm{g}}^{\mathrm{si}}}{r_{\mathrm{d} . \mathrm{g}}^{\mathrm{so}}}
$$

Substituting (11) into (10), after some transformations, we derive

$$
q=\frac{\lambda}{\delta}\left(t_{\mathrm{so}}-t_{\mathrm{si}}\right) \frac{\left(r_{\mathrm{d} . \mathrm{g}}^{\mathrm{si}} / r_{\mathrm{d} . \mathrm{g}}^{\mathrm{so}}\right)^{A}}{\left(r_{\mathrm{d} . \mathrm{g}}^{\mathrm{si}} / r_{\mathrm{d} . \mathrm{g}}^{\mathrm{so}}\right)^{A}-1} \ln \left(r_{\mathrm{d} . \mathrm{g}}^{\mathrm{si}} / r_{\mathrm{d} . \mathrm{g}}^{\mathrm{so}}\right)^{A},
$$

where $A=D\left(\mu_{\mathrm{s}} c_{\mathrm{s}}\right)_{p} /\left(a \mu c_{p}\right)$.

In the absence of the mass transfer, i.e., at $r_{\mathrm{d} . \mathrm{g}}^{\mathrm{so}}=r_{\mathrm{d} . \mathrm{g}}^{\mathrm{si}}$, taking into account that $\rho_{\mathrm{s}} / \rho=\mu_{\mathrm{s}} / \mu$,

$$
q_{\lambda}=\frac{\lambda}{\delta}\left(t_{\mathrm{so}}-t_{\mathrm{si}}\right)=\frac{a \rho c_{p}}{\delta}\left(t_{\mathrm{so}}-t_{\mathrm{si}}\right) .
$$

After the termwise division of Eq. (12) by (13), the coefficient that takes into account the influence of the Stefan flow on the heat emission coefficient, is calculated by the formula

$$
K_{q}=\frac{q}{q_{\lambda}}=\frac{\left(r_{\mathrm{d} . \mathrm{g}}^{\mathrm{si}} / r_{\mathrm{d} . \mathrm{g}}^{\mathrm{so}}\right)^{A}}{\left(r_{\mathrm{d} . \mathrm{g}}^{\mathrm{si}} / r_{\mathrm{d} . \mathrm{g}}^{\mathrm{so}}\right)^{A}-1} \ln \left(r_{\mathrm{d} . \mathrm{g}}^{\mathrm{si}} / r_{\mathrm{d} . \mathrm{g}}^{\mathrm{so}}\right)^{A} .
$$

If complex $\left(r_{\mathrm{d} . \mathrm{g}}^{\mathrm{si}} / r_{\mathrm{d} . \mathrm{g}}^{\mathrm{so}}\right)^{A}$ does not strongly differ from unity, then, expanding the logarithm into the series,

$$
\ln x=\frac{x-1}{x}+\frac{1}{2}\left(\frac{x-1}{x}\right)^{2}+\ldots
$$

and limiting ourselves by first two terms, we derive the approximated formula

$$
K_{q} \approx 1+\frac{1}{2} \frac{\left(r_{\mathrm{d} . \mathrm{g}}^{\mathrm{si}} / r_{\mathrm{d} . \mathrm{g}}^{\mathrm{so}}\right)^{A}-1}{\left(r_{\mathrm{d} . \mathrm{g}}^{\mathrm{si}} / r_{\mathrm{d} . \mathrm{g}}^{\mathrm{so}}\right)^{A}}=1+\frac{1}{2}\left[1-\left(r_{\mathrm{d} . \mathrm{g}}^{\mathrm{si}} / r_{\mathrm{d} . \mathrm{g}}^{\mathrm{so}}\right)^{A}\right],
$$

We can clearly see from this formula that the heat flow also increases as the "accompanying" diffusion flow increases.

After similar calculations for the case when the heat and mass flows are directed oppositely $\left(t_{\mathrm{so}}<t_{\mathrm{si}}\right)$, the formula for the calculation of coefficient $K_{q}$ takes the form

$$
K_{q}=\frac{\ln \left(r_{\mathrm{d} . \mathrm{g}}^{\mathrm{si}} / r_{\mathrm{d} . \mathrm{g}}^{\mathrm{so}}\right)^{A}}{\left(r_{\mathrm{d} . \mathrm{g}}^{\mathrm{si}} / r_{\mathrm{d} . \mathrm{g}}^{\mathrm{so}}\right)^{A}-1} .
$$

If complex $\left(r_{\mathrm{d} . \mathrm{g}}^{\mathrm{si}} / r_{\mathrm{d} . \mathrm{g}}^{\mathrm{so}}\right)^{A}$ does not strongly differ from unity, then, expanding the logarithm into the same series and limiting ourselves by the first term, we derive $K_{q} \approx\left(r_{\mathrm{d} . \mathrm{g}}^{\mathrm{so}} / r_{\mathrm{d} . \mathrm{g}}^{\mathrm{si}}\right)^{A}$. Since ratio $r_{\mathrm{d} . \mathrm{g}}^{\mathrm{so}} / r_{\mathrm{d} . \mathrm{g}}^{\mathrm{si}}$ is smaller than unity, the heat flow decreases as the diffusion counter flow decreases. 


\section{HEAT-AND-MASS TRANSFER WITH THE SPHERE IN AN INFINITE MEDIUM}

In this section, we consider another canonic case of the heat transfer, namely, the evaporation from the spherical surface (the source), which is placed into an infinite gas mixture in the absence of the natural and forced convection when the temperature of this surface $t_{\mathrm{so}}$ is higher than surrounding medium temperature $t_{\mathrm{si}}$, while the heat flow density on the sphere surface is $q_{\mathrm{so}}$. The equation for the calculation of the steam flow density on sphere surface $j_{\mathrm{s}}^{\text {so }}$ allowing for (4) has the form

$$
\frac{d Y}{Y^{2}}=-\frac{D}{j_{\mathrm{s}}^{\mathrm{s}} \bar{V}_{\mu} \delta} \frac{d r_{\mathrm{s}}}{\left(1-r_{\mathrm{s}}\right)}=\frac{D}{j_{\mathrm{s}}^{\mathrm{s} 0} \bar{V}_{\mu} \delta} \frac{d\left(1-r_{\mathrm{s}}\right)}{\left(1-r_{\mathrm{s}}\right)},
$$

where $Y=y / \delta$ is dimensionless radius, $y$ is the radiusvector (in order not to confuse with the relative volume concentration $r$ ), $\delta$ is the sphere radius (it is denoted similarly to the reduced film thickness for obtaining the identical dependences), $j_{\mathrm{s}}^{\text {so }}$ is the steam flow density on the sphere surface, and $j_{\mathrm{s}}=j_{\mathrm{s}}^{\text {so }} / Y^{2}$. The steam flow density relative to the motionless dry gas (correspondingly, the motionless sphere surface) is expressed by formula (4) similarly to the planar problem. Since the dry gas is motionless, then the densities of the steam and heat flows should be inversely proportional to the square of the radius-vector size.

The solution of this equation at boundary condition $r_{\mathrm{s}}=r_{\mathrm{s}}^{\mathrm{si}}$, when $Y \rightarrow \infty$,

$$
\frac{1}{Y}=\frac{D}{j_{\mathrm{s}}^{\mathrm{s} 0} \bar{V}_{\mu} \delta} \ln \frac{1-r_{\mathrm{s}}^{\mathrm{si}}}{1-r_{\mathrm{s}}} .
$$

The steam concentration on the sphere surface $(Y=1)$ equals $r_{\mathrm{s}}^{\mathrm{so}}$. When substituting this condition, Eq. (15) is transformed in (6), in which $j_{\mathrm{s}}$ is substituted for $j_{\mathrm{s}}^{\mathrm{so}}$.

Specific heat flow $q$, which passes through any spherical surface with temperature $t$ and radius $Y$, is described by Eq. (8) as before. Substituting $j_{\mathrm{s}}=j_{\mathrm{s}}^{\text {so }} / Y^{2}$ and $q=q_{\mathrm{so}} / Y^{2}$, we substitute the differential equation with separable variables

$$
\frac{q_{\mathrm{so}}}{Y^{2}}=-\frac{\lambda}{\delta} \frac{d t}{d Y}+\frac{j_{\mathrm{s}}^{\mathrm{so}}\left(\mu_{\mathrm{s}} c_{\mathrm{s}}\right)_{p}}{Y^{2}}\left(t-t_{\mathrm{si}}\right),
$$

where $q_{\text {so }}$ is the heat flow density.

Substituting $\theta=\left(t-t_{\mathrm{si}}\right) /\left(t_{\mathrm{so}}-t_{\mathrm{si}}\right)$ and $\mathrm{Sf}=$ $j_{\mathrm{s}}^{\mathrm{so}}\left(\mu_{\mathrm{s}} c_{\mathrm{s}}\right)_{p} \delta / \lambda$, we transform this equation:

$$
\frac{d Y}{Y^{2}}=\frac{d \theta}{\operatorname{Sf} \theta-\frac{q_{\mathrm{so}} \delta}{\lambda\left(t_{\mathrm{so}}-t_{\mathrm{si}}\right)}}
$$

The formula for the heat flow, which is derived from its solution with boundary conditions $\theta=1$ at
$Y=1$ and $\theta=0$ at $Y \rightarrow \infty$, coincides with formula (12), if we substitute $q_{\mathrm{so}}$ and $j_{\mathrm{s}}^{\text {so }}$ instead of $q$ and $j_{\mathrm{s}}$ into the latter. Correspondingly, the ratio of specific heat flows from the sphere surface in the presence and in the absence of the mass transfer $\left(K_{q}=q_{\mathrm{so}} / q_{\lambda \text {, so }}\right)$ will be determined by formula (14).

This coincidence of solutions from two different geometric conditions allows us to assume that the influence of the mass transfer on the heat exchange upon flowing around the bodies by the steam-gas mixture flow can be also evaluated by coefficient $K_{q}$ calculated by formula (14).

The validity of this assumption was also confirmed by Leont'ev [7], where the mass transfer and gas injection/suction at $D=a$ are analyzed for the boundary layer, which is formed during the longitudinal flowing around the plate, while the derived relationships describe well the results found for flowing around other bodies, as it is seen from the figure presented in [7].

According to formula (14), the influence of the mass transfer on the heat emission coefficient is determined by complex $\left(r_{\mathrm{d} . \mathrm{g}}^{\mathrm{si}} / r_{\mathrm{d} . \mathrm{g}}^{\mathrm{so}}\right)^{\frac{D}{a}} \frac{\left(\mu_{\mathrm{s}} c_{\mathrm{s}}\right)_{p}}{\mu c_{p}}$. As the latter increases, the heat emission coefficient increases if the mass and heat flows are unidirectional and decreases if the flows are directed oppositely.

Based on Eq. (14), we can evaluate the possible increase in the heat emission coefficient during the deep heat utilization of the burning products of the natural gas in condensation boilers. Let us accept $r_{\mathrm{d} . \mathrm{g}}^{\mathrm{so}}=0.814 ; r_{\mathrm{d} . \mathrm{g}}^{\mathrm{si}}=0.987 ; t_{\mathrm{so}}=100^{\circ} \mathrm{C} ; t_{\mathrm{si}}=10^{\circ} \mathrm{C} ;$ $\left(\mu_{\mathrm{s}} c_{\mathrm{s}}\right)_{p}=34.6 \mathrm{~kJ} /(\mathrm{kmol} \mathrm{K}), \mu c_{p}=30.16 \mathrm{~kJ} /(\mathrm{kmol} \mathrm{K})$ (at $\left.100^{\circ} \mathrm{C}\right) ; D / a=1.17$. The calculation by formula (14) for these conditions gives an increase in the heat transfer coefficient due to the Stefan flow by $13.5 \%$ ( $11.4 \%$ by the approximate formula).

\section{ACCOUNT FOR THE PHASE TRANSITION HEAT}

The more substantial increase in the heat emission coefficient in the condensation boiler is provided due to phase-transition heat $\Delta h_{\mathrm{p} . \mathrm{t}}$. If correction $K_{j}$, which is calculated by formula (7), is also valid for the cases of the convective heat-and-mass transfer, we can easily calculate the mass steam flow, which condensates on the surface, by this formula using the analogy between the heat-and-mass transfer processes.

Summary heat flow density $q_{\Sigma}$ on the surface is summed of heat flow $q$, which is determined by expression (14) in which $q_{\lambda}$ is counted for the case of "dry" heat transfer in the absence of the Stefan flow and the heat liberated during steam condensation $j_{\mathrm{s}} \Delta h_{\mathrm{p} . \mathrm{s}}$. Steam flow, $\mathrm{kg} /\left(\mathrm{m}^{2} \mathrm{~s}\right)$, is calculated by formula $j_{\mathrm{s}}=K_{j} j_{\mathrm{s}}^{0}=K_{j} \beta \rho_{\mathrm{s}}\left(r_{\mathrm{s}}^{\mathrm{so}}-r_{\mathrm{s}}^{\mathrm{si}}\right)$, where mass output coeffi- 
cient $\beta$ is found by formula $\mathrm{Nu}_{\mathrm{d}}=f\left(\operatorname{Re}, \mathrm{Pr}_{\mathrm{d}}\right)$, which is similar to known dependence $\mathrm{Nu}_{\lambda}=f(\mathrm{Re}, \mathrm{Pr})$ for the dry heat transfer. For example, it is recommended to calculate the heat transfer during the transverse flowing around the depth rows of the staggered pipe bundle at $\mathrm{Re}=10^{3}-10^{5}$ by formula $\mathrm{Nu} \cong 0.41 \operatorname{Re}^{0.6} \operatorname{Pr}^{0.33}$ [5]. For tubes $38 \mathrm{~mm}$ in diameter in the bundle flown round by air with the velocity of $10 \mathrm{~m} / \mathrm{s}$ and the temperature of $100^{\circ} \mathrm{C}\left[\lambda=3.18 \times 10^{-2} \mathrm{~W} /(\mathrm{m} \mathrm{K}), \nu=23.5 \times\right.$ $\left.10^{-6} \mathrm{~m}^{2} / \mathrm{s}, \operatorname{Pr}=0.69\right]$, heat transfer coefficient $\alpha_{\lambda}=$ $101.7 \mathrm{~W} /\left(\mathrm{m}^{2} \mathrm{~K}\right)$. Taking into account the correction by formula (14) at $r_{\mathrm{d} . \mathrm{g}}^{\mathrm{so}}=0.814$ and $r_{\mathrm{d} . \mathrm{g}}^{\mathrm{si}}=0.987$, we find $\alpha=115.5 \mathrm{~W} /\left(\mathrm{m}^{2} \mathrm{~K}\right)$. Allowing for the correction by (7),

$$
\begin{gathered}
K_{j} \beta=K_{j}\left(\mathrm{Nu}_{\mathrm{d}} \frac{D}{d}\right)=1.114 \times 0.117=0.13 \mathrm{~m} / \mathrm{s} ; \\
j_{\mathrm{s}}=K_{j} \beta \rho_{\mathrm{s}}\left(r_{\mathrm{s}}^{\mathrm{so}}-r_{\mathrm{s}}^{\mathrm{si}}\right)=0.13 \times 0.589(0.987-0.814) \\
=0.0132 \mathrm{~kg} /\left(\mathrm{m}^{2} \mathrm{~s}\right) .
\end{gathered}
$$

At $\Delta h_{\mathrm{p} . \mathrm{t}}=2477 \mathrm{~kJ} / \mathrm{kg}, q_{\text {cond }}=j_{\mathrm{s}} \Delta h_{\mathrm{p} . \mathrm{t}}=0.0132 \times$ $2477=32.7 \mathrm{~kW} / \mathrm{m}^{2}$, while summary heat flow $q_{\Sigma}=$ $\alpha\left(t_{\mathrm{so}}-t_{\mathrm{si}}\right)+q_{\mathrm{cond}}=115.5(100-10) 10^{-3}+32.7=$ $43.1 \mathrm{~kW} / \mathrm{m}^{2}$.

\section{CONCLUSIONS}

(1) When solving the problems on the influence of the Stefan flow on the heat transfer coefficient with the accompanying heat-and-mass transfer through a planar reduced film and from the spherical surface in an infinite medium, identical relationships are derived. This makes it possible to conclude the independence of this influence on the geometric characteristics of the heat-transfer surfaces.
(2) The phase transition heat in the presented example gives a more substantial contribution to the summary heat emission coefficient compared with the convective component of the heat flow. Therefore, an increase in the steam transfer from the steam-gas mixture to the surface under the effect of the Stefan flow leads to a more substantial increase in the condensation component compared with the convective one.

\section{ACKNOWLEDGMENTS}

We thank E.M. Tolmachev for useful discussions.

\section{REFERENCES}

1. L. D. Landau and E. M. Lifshits, Mechanics of Continuous Media (GITTL, Moscow, 1954) [in Russian].

2. D. A. Frank-Kamenetskii, Diffusion and Heat Transfer in the Chemical Kinetics (Nauka, Moscow, 1967) [in Russian].

3. T. K. Sherwood, R. L. Pigford, R.L., and C. R. Wilke, McGraw-Hill Engineering Series: Mass Transfer (McGraw-Hill, Tokyo, 1978).

4. R. Haaze, Thermodynamics of Irreversible Processes (Mir, Moscow, 1967) [in Russian].

5. V. P. Isachenko, V. A. Osipova, and A. S. Sukomel, Heat Transfer (Energoizdat, Moscow, 1981) [in Russian].

6. A. P. Baskakov, "Effect of the Stefan Flux on Coefficients of Heat- and Mass Transfer (a Binary System)," Therm. Eng. 59 (7), 544-549 (2012).

7. A. I. Leont'ev, "Engineering methods for calculating friction and heat transfer on an impermeable surface," Teploenergetika, No. 9, 19-24 (1972).

Translated by N. Korovin 